\title{
Facilitadores y barreras percibidos en la práctica de la actividad física en adolescentes escolarizados en Piedecuesta (Santander), en 2016: análisis cualitativo
}

Perceived facilitators and barriers to physical activity in adolescents in Piedecuesta (Santander), in 2016: qualitative analysis

\section{Facilitadores e barreiras evidentes na prática da atividade física nos adolescentes em idade escolar em Piedecuesta Santander, em 2016: Análise qualitativa}

Johanna Otero; Daniel Dylan Cohen²; Diana María Delgado-Chinchilla; Paul Anthony Camacho-López;; Mónica Andrea Amador-Ariza5; Sandra Milena Rueda-Quijano6; Patricio López-Jaramillo?.

1 MSc en Salud Pública, Universidad de Santander (UDES). Bucaramanga, Santander (Colombia), Fundación Oftalmológica de Santander (FOSCAL). Floridablanca, Santander. jaoterow@gmail.com. ORCID: https://orcid.org/0000-0002-2044-2071.

2 PhD en Ciencias del Ejercicio, uDEs. Bucaramanga, Santander (Colombia). danielcohen1971@gmail.com. oRCiD: https://orcid.org/00000002-0899-4623.

3 Máster en Desarrollo Social, FosCAL. Floridablanca, Santander (Colombia). dianadelchi@gmail.com oRCID: https://orcid.org/0000-00026312-4604.

4 MSc en Epidemiología, FosCAL. Floridablanca, Santander (Colombia), Universidad Autónoma de Bucaramanga (UNAB), Floridablanca, Santander. paul.camacho@foscal.com.co. oRcil: https://orcid.org/0000-0002-6233-9582.

5 Fisioterapeuta, UDES. Bucaramanga, Santander (Colombia). monicaamador1@hotmail.com. oRCID: https://orcid.org/0000-0002-2879-4813.

6 Médica, UnAB, Floridablanca, Santander (Colombia).srueda108@unab.edu.co. oRcID: https://orcid.org/0000-0003-0811-2173.

7 PhD en Ciencias Biológicas y Farmacología, udes. Bucaramanga, Santander (Colombia), FosCAL. Floridablanca, Santander. jplopezj@ gmail.com. ORCID: https://orcid.org/0000-0002-9122-8742.

Recibido: 26/03/2019. Aprobado: 19/12/2019. Publicado: 20/03/2020

Otero J, Cohen DD, Delgado-Chinchilla DM, Camacho-López PA, Amador-Ariza MA, Rueda-Quijano SM, López-Jaramillo P. Facilitadores y barreras percibidos en la práctica de la actividad física en adolescentes escolarizados en Piedecuesta (Santander), en 2016: análisis cualitativo. 2020;38(2):e337834. DOI: https://doi.org/10.17533/udea.rfnsp.e337834

\section{Resumen}

Objetivo. Describir la percepción que tienen adolescentes escolarizados entre 12 y 16 años, en Piedecuesta (Santander), en 2016, sobre los facilitadores y las barreras que influyen en la práctica de la actividad física. Métodos. Análisis cualitativo desde un enfoque fenomenológico, con la participación de
20 adolescentes escolarizados que, mediante entrevistas semiestructuradas y un grupo focal, conversaron sobre la práctica, los beneficios, los entornos y la influencia y la compañía en relación con la actividad física, para identificar barreras y facilitadores. Resultados. La práctica de la

* Este artículo surge de la investigación "Fuerza muscular y capacidad aeróbica en adolescentes escolares con bajo peso al nacer y riesgo metabólico" (SIMAC), realizada en Piedecuesta (Santander), en 2016. SIMAC fue registrado en Clinical Trials NTC03779737 (https://clinicaltrials. gov/ct2/show/NCT03779737). Fecha de terminación: diciembre de 2018. 
actividad física se limitaba a las clases de educación física en la mayoría de los casos. El compañero principal en la práctica extracurricular era el padre del mismo sexo. La actividad física representaba una oportunidad para el disfrute, mejorar la salud física y mental, así como la contextura corporal, y para romper con la rutina diaria. La falta de tiempo por compromisos académicos fue la razón principal para no realizar actividad física. Los lugares públicos no siempre estaban disponibles, a menudo no se consideraban seguros. En la escuela, el espacio y las instalaciones eran inadecuados, y el uso estaba restringido. Conclusiones. La escuela es un punto de convergencia importante para la práctica de la actividad física, lo que sugiere la necesidad de fortalecer el plan de estudios al respecto; este entorno es un facilitador potencial para aumentar el nivel de actividad física en adolescentes. También se percibe como barrera, dado que la práctica interfiere con las actividades académicas. Las mejoras en la seguridad y el atractivo estético en el entorno comunitario se visibilizan como un facilitador para promover la actividad física.

--Palabras clave: Actividad física; educación física; estilo de vida saludable; adolescente; Piedecuesta, Santander (Colombia).

\section{Abstract}

Objective: Describe the perception of adolescents aged between 12 and 16 years, in Piedecuesta (Santander), in 2016, about the facilitators and barriers that influence physical activity. Methodology:. Qualitative analysis from a phenomenological approach, with the participation of 20 adolescents who talked about the practice, benefits, environments, influence and company in connection with physical activity, to identify barriers and facilitators, through semi-structured interviews and a focus group. Results: Physical activity was limited to physical education classes in most cases. The main companion in extracurricular physical activity was the parent of the same sex. Physical activity represented an opportunity for enjoyment, improvement of physical and mental health, as well as body composition, and to have a break in their daily routine.
Lack of time due to academic commitments was the main reason for not doing physical activity. Public places were not always available and they were often not considered to be safe. Space and facilities at school were inadequate, and their use was restricted. Conclusions: The school is an important point of convergence for doing physical activity, suggesting the need to strengthen the curriculum in this respect; this environment is a potential facilitator to increasing the level of physical activity in adolescents. It can also be seen as a barrier, given that the practice interferes with academic activities. Improvements in safety and aesthetic appeal in the surrounding community are seen as a facilitator to promoting physical activity.

---------Key words: Physical activity; physical education; healthy life style; adolescent; Piedecuesta, Santander (Colombia)

\section{Resumo}

Objetivo: Descrever a percepção que os adolescentes entre 12 e 17 anos de Piedecuesta em Santander, tiveram em 2016 sobre os facilitadores e as barreiras que influenciaram na prática da atividade física. Metodologia: Análise qualitativa partindo de um foco fenomenológico, com a participação de 20 adolescentes em idade escolar que, através de entrevistas semiestruturadas e um grupo focal, conversaram sobre a prática, os benefícios, os entornos, a influência e o acompanhamento relacionados com a atividade física, para identificar barreiras e facilitadores. Resultados: Na maioria dos casos, a prática da atividade física limitava-se às aulas de educação física. $\mathrm{O}$ companheiro principal na prática extracurricular era o genitor ou genitora do mesmo sexo. A atividade física representava uma oportunidade para disfrutar, melhorar a saúde física e mental, além da estrutura corporal e para sair da rotina diária. A falta de tempo devido às responsabilidades escolares foi a principal razão para não realizarem atividade física. Os lugares públicos nem sempre estavam disponíveis e geralmente não são considerados seguros. Na escola, o espaço e as instalações eram inadequados e o uso estava restrito. Conclusões: A escola é um importante ponto de convergência para a prática da atividade física, o que determina a necessidade de fortalecer o currículo relacionado; esse ambiente é um facilitador potencial para aumentar o nível de atividade física nos adolescentes. Também se percebe como barreira, já que a prática interfere com as atividades escolares. As melhorias na segurança e um espaço comunitário mais atraente do ponto de vista estético são determinantes como facilitadores para a promoção da atividade física.

---------Palavras-chave: Atividade física; educação física; estilo de vida saudável; adolescente; Piedecuesta; Santander (Colômbia). 


\section{Introducción}

Los estilos de vida en la cultura occidental y el fenómeno de urbanización han alentado a diferentes grupos de población, incluidos los adolescentes, a acrecentar el consumo de alimentos azucarados y grasos, y el sedentarismo [1]. La inactividad física es un contribuyente importante en el desarrollo de enfermedades no transmisibles en los países de ingresos altos, y está aumentando en los de ingresos medios y bajos [2]. Estos factores de riesgo modificables se asocian con el incremento en la prevalencia de obesidad en jóvenes y en el riesgo de desarrollar enfermedades cardiometabólicas en la edad adulta [3]. Algunos investigadores han sugerido que el sexo, la edad y los grupos socioculturales son determinantes en los adolescentes que optan por vidas cada vez más sedentarias e inactivas [4]. En la actualidad, las estrategias para promover un aumento en la práctica de la actividad física (AF) constituyen una prioridad para la salud pública; esto es particularmente significativo en países de ingresos bajos y medios, donde la carga de morbilidad y mortalidad por enfermedades crónicas no transmisibles es mayor [5].

Aproximadamente, el $81 \%$ de los adolescentes (13 a 15 años) en todo el mundo no siguen recomendaciones internacionales de práctica de actividad física, como la realización diaria de al menos 60 minutos [6-8]. También se ha documentado que el tiempo dedicado a la $\mathrm{AF}$ disminuye con la edad y que los niños son más activos que las niñas [9]. Las recomendaciones internacionales de ejercicio para adultos (de 18 a 64 años) son de al menos 150 minutos de AF de intensidad moderada, al menos 75 minutos de AF de intensidad vigorosa o una combinación equivalente de actividad de intensidad moderada y vigorosa por semana [6]. En Colombia, el $56,6 \%$ de los adultos entre los 18 y 29 años cumplen esta recomendación mediante actividades de tiempo libre y de transporte activo [10]. En una encuesta de 2011 sobre AF (tiempo libre, trabajo y transporte) en Bucaramanga, Colombia, niveles bajos de esta fueron observados en dos tercios de los jóvenes (de 15 a 24 años), y en un porcentaje significativamente mayor de mujeres $(79,5 \%$ mujeres vs. 48,5 \% hombres) [11]. Para que los niveles de AF continúen desde la niñez hasta la edad adulta [12], es necesario fortalecer el establecimiento de buenos hábitos de AF en la adolescencia y juventud, en aras de lograr el respaldo de los objetivos de la AF en la vida posterior, entre ellos, como lo ya mencionado, el cuidado de la salud cardiovascular.

Varios autores han sugerido que las razones por las cuales las personas participan o abandonan la $\mathrm{AF}$ difieren según la edad, y están en relación con el tipo y la frecuencia de la $\mathrm{AF}$ en la que participa normalmente la población [13-15]. Algunos autores también argumentan que las variables sociales, como el cumplimiento de las normas y el apoyo social de las redes familiares y de amistad, son más importantes que la influencia del entorno [16]. En este contexto, el juego y el transporte activo se convierten en las mejores oportunidades para participar en AF $[17,18]$. Entre los datos que describen los patrones de AF de los jóvenes en Colombia y otros países con diferente categorización por ingresos [10,19], existe la necesidad de mejorar la comprensión de los determinantes de la práctica de la $\mathrm{AF}$ en adolescentes en el contexto, para informar y aportar en el diseño de estrategias para aumentar el nivel de participación desde una edad temprana.

El presente artículo muestra los resultados de un análisis cualitativo en el marco del proyecto "Fuerza muscular y capacidad aeróbica en adolescentes escolares con bajo peso al nacer y riesgo metabólico", denominado SIMAC. Este fue diseñado para evaluar la condición física y los cambios antropométricos producidos en los adolescentes por una intervención de AF extracurricular.

Este proyecto fue un ensayo clínico controlado, implementado en adolescentes de una institución educativa oficial en Piedecuesta (Santander, Colombia), municipio perteneciente al área metropolitana de Bucaramanga, que ocupa el quinto lugar, de acuerdo con la densidad poblacional, entre los 87 municipios del departamento. El colegio elegido matricula anualmente alrededor de 1700 estudiantes de secundaria; así, ocupa el tercer lugar entre los de categoría oficial con sede en Piedecuesta.

El objetivo de aplicar métodos cualitativos fue describir la percepción que tienen adolescentes escolarizados entre 12 y 16 años, en Piedecuesta (Santander), en 2016, inscritos en SIMAC, sobre los facilitadores y las barreras que influyen en la práctica de la AF.

\section{Metodología}

\section{Diseño de la investigación}

El uso de una metodología cualitativa es relevante, ya que permite comprender las perspectivas de los jóvenes sobre la AF, así como los factores clave, según ellos, que se convierten en "facilitadores" y "barreras" para la participación en AF [20]. De esta manera, dicha metodología complementa la evidencia cuantitativa para proporcionar mejores herramientas para la planificación de las intervenciones de salud pública.

El estudio fue desarrollado desde la perspectiva fenomenológica, es decir, mediante la exploración y la descripción de las experiencias de adolescentes escolarizados, para la comprensión de facilitadores 
y barreras implicados en la práctica de la AF según la percepción de ellos. Fue realizado con participantes del estudio SIMAC, desarrollado en las instalaciones de un centro público de recreación ubicado en Piedecuesta, Santander (Colombia).

Durante 2016, el estudio SIMAC reclutó 244 adolescentes entre 12 y 17 años, de los cuales 111 fueron asignados al azar a dos grupos de intervención, ya fuera de actividades aeróbicas y de entrenamiento de fuerza, o a un grupo control.

El Comité de Ética e Investigación de la Fundación Oftalmológica de Santander (FOSCAL) aprobó el estudio mediante Acta 39 del 19 de febrero de 2016, y todos los adolescentes incluidos expresaron su interés en participar, y sus padres otorgaron consentimiento escrito.

\section{Recolección de datos}

La selección de los participantes se hizo según el orden del código asignado aleatoriamente para las intervenciones del estudio SIMAC. Se garantizó que fueran seleccionados estudiantes de todos los grados académicos, en igual número de hombres y mujeres, y que contaran con el consentimiento diligenciado y autorizado por escrito.

Luego, los estudiantes fueron llamados vía telefónica para preguntar sobre su disposición y disponibilidad para participar tanto en una entrevista individual como en un grupo focal. Invitamos a 20 adolescentes a participar en la entrevista y a 7 en el grupo focal, actividades que se realizaron en una sola sesión durante la fase de familiarización de SIMAC, antes de comenzar la intervención.

Los padres completaron un cuestionario para definir las características sociodemográficas generales, incluidos los indicadores del nivel de ingresos, como afiliación al sistema de salud, el estrato de la vivienda, la situación laboral del padre o madre de cabeza de hogar, el ingreso mensual promedio del hogar (en Colombia, en 2016, equivalente a aproximadamente a $\$ 767000$ ) y el tipo de familia (nuclear: madre y padre que viven juntos con el adolescente, y otro).

Un investigador del estudio SIMAC, con perfil de salubrista, y un asistente de investigación (comunicadora social) condujeron las entrevistas y el grupo focal; ninguno de los dos estuvo involucrado en la implementación de los protocolos del estudio, dado que no pertenecían al grupo de implementación de la estrategia de intervención de SIMAC. Cada entrevista tuvo una duración entre 40 y 60 minutos, mientras que el grupo focal se condujo por aproximadamente 2 horas.

En ambos abordajes, fueron tratados los siguientes temas con relación a la experiencia y la percepción de la AF: definición, práctica, beneficios, entornos, e influencia y compañía.
Las entrevistas y el grupo focal fueron grabados. Se realizaron transcripciones de los audios y se unificaron con notas tomadas por el asistente del grupo focal.

\section{Análisis de datos}

Las variables sociodemográficas de los participantes se extrajeron de la base de datos de referencia del sIMAC. Dos investigadores leyeron de forma independiente los textos transcritos y las notas de campo, para identificar conceptos emergentes no preconcebidos. No hubo restricción en la codificación inicial, y se identificaron todos los conceptos emergentes.

El software NVivo Pro ${ }^{\circledR}$ (QSR International®). Melbourne, Australia; versión 11 para Windows) se utilizó para la codificación y la identificación de nodos. Después de la codificación, fue revisado el mapa de nodos y, por consenso entre los investigadores, se agrupó en subtemas y temas.

Las perspectivas de los participantes se exploraron completamente en términos de amplitud (contraste de dos o más perspectivas) y profundidad (visión de una sola perspectiva).

La transcripción y el análisis de grupos focales permitió identificar 30 nodos, y la de las entrevistas, entre 27 y 47 nodos. Para el caso de los "facilitadores", se identificaron como principales nodos la salud como beneficio, la compañía e influencia de los padres, los centros recreativos y parques como espacios para hacer AF, y como motivación, la diversión o distracción. Por su parte, como "barreras", los principales nodos identificados, de acuerdo con el número de referencias, fueron para el abandono de la práctica de la $\mathrm{AF}$, los compromisos académicos y la falta de tiempo, y tanto el barrio como el colegio en lo referente a espacios para la práctica.

Finalmente, los temas se buscaron en diferentes buscadores como palabras clave en la literatura disponible, para agotar todos los temas relevantes y evaluar la consistencia de los hallazgos.

\section{Resultados}

Fueron incluidos 20 participantes con igual distribución por sexo, con 14,3 $\pm 1,1$ años de edad. Hubo participación de al menos un adolescente por grado académico (séptimo a décimo).

El 45,0\% de los participantes no estaba afiliado al sistema de salud o pertenecía a régimen subsidiado; sin embargo, el 90,0\% de los padres era activo laboralmente.

El $10,0 \%$ de los hogares percibía en promedio menos de un salario mínimo al mes. El 55,0 \% de los adolescentes residía en viviendas de estrato bajo. Y tres cuartas partes de los participantes pertenecían a familias nucleares (véase tabla 1 ). 
Tabla 1. Características sociodemográficas de los participantes

\begin{tabular}{|c|c|c|c|c|c|c|c|}
\hline \multirow{2}{*}{\multicolumn{2}{|c|}{ Características de los participantes }} & \multicolumn{2}{|c|}{ Mujeres } & \multicolumn{2}{|c|}{ Hombres } & \multicolumn{2}{|c|}{ Total } \\
\hline & & $\mathrm{n}$ & $\%$ & $\mathrm{n}$ & $\%$ & $\mathrm{n}$ & $\%$ \\
\hline \multirow{5}{*}{$\begin{array}{l}\text { Grupos de edad } \\
\text { (años) }\end{array}$} & 12 & 1 & 10,0 & 0 & 0,0 & 1 & 5,0 \\
\hline & 13 & 1 & 10,0 & 2 & 20,0 & 3 & 15,0 \\
\hline & 14 & 3 & 30,0 & 5 & 50,0 & 8 & 40,0 \\
\hline & 15 & 5 & 50,0 & 1 & 10,0 & 6 & 30,0 \\
\hline & 16 & 0 & 0,0 & 2 & 20,0 & 2 & 10,0 \\
\hline \multirow{2}{*}{$\begin{array}{l}\text { Afiliación al sistema de } \\
\text { salud }\end{array}$} & No afiliado o subsidiado & 3 & 30,0 & 6 & 60,0 & 9 & 45,0 \\
\hline & Contributivo o especial & 7 & 70,0 & 4 & 40,0 & 11 & 55,0 \\
\hline \multirow{2}{*}{$\begin{array}{l}\text { Situación laboral (jefe } \\
\text { de hogar) }\end{array}$} & Desempleado/estudiante/ama de casa & 1 & 10,0 & 1 & 10,0 & 2 & 10,0 \\
\hline & Empleado/independiente/retirado & 9 & 90,0 & 9 & 90,0 & 18 & 90,0 \\
\hline \multirow{2}{*}{$\begin{array}{l}\text { Ingreso mensual } \\
\text { promedio del hogar }\end{array}$} & $\leq 1$ salario mínimo & 2 & 20,0 & 0 & 0,0 & 2 & 10,0 \\
\hline & $>1$ salario mínimo & 8 & 80,0 & 10 & 100 & 18 & 90,0 \\
\hline \multirow{2}{*}{ Estrato de vivienda } & Bajo & 5 & 50,0 & 6 & 60,0 & 11 & 55,0 \\
\hline & Medio & 5 & 50,0 & 4 & 40,0 & 9 & 45,0 \\
\hline \multirow{2}{*}{ Tipo de unidad familiar } & Nuclear & 7 & 70,0 & 8 & 80,0 & 15 & 75,0 \\
\hline & Otro & 3 & 30,0 & 2 & 20,0 & 5 & 25,0 \\
\hline
\end{tabular}

A continuación, los temas se muestran en orden descendente de acuerdo con su peso porcentual. Los testimonios de los participantes se presentan por sexo (Hombre: H, y Mujer: M) y por edad en años.

\section{Conceptualización y práctica}

Los adolescentes definieron la AF como un medio para hacer ejercicio, facilitar el movimiento corporal y crear momentos recreativos. El concepto estuvo asociado con los deportes y con la vida diaria en actividades al aire libre, como saltar o trotar.

Es el movimiento de los cuerpos, haciendo una fuerza, hacer ejercicio. Cuando uno corre, cuando alza pesas, cuando caminas, cuando monta cicla (H, 14 años).

Estar ejercitándome para poder tener un buen ritmo, salir a trotar o a correr, o hacer estiramiento en la casa. Un buen ritmo: salir a caminar. Ejercitarse: ir a un gimnasio a hacer pesas o cardio (M, 15 años).

Su práctica se limitó a las clases de educación física; pocos adolescentes informaron que dedican tiempo a actividades recreativas extracurriculares (juegos con pelota para lanzar o patear en parques, o caminatas) o actividades deportivas (específicamente, patinaje, baloncesto o fútbol).

Dos horas de actividad física a la semana, solo las de la clase de educación física (H, 13 años).

[...] en las clases del colegio, dos horas por semana. La mayoría de las clases estoy en constante movimiento, corriendo. Me gusta y es muy importante asistir a la clase (H, 14 años).

\section{Facilitadores}

Los padres, principalmente sus pares por sexo, eran los principales compañeros de los adolescentes en la práctica de la $\mathrm{AF}$ fuera de la escuela.

Con mi mami. No le gusta salir sola, prefiero salir con ella. No con mi hermano o el papá, porque trabajan muy temprano. La mayoría de los amigos estudian en la mañana y no pueden. El resto de la familia vive lejos. Con mi mamá vamos hablando y disfruto de ese momento (M, 15 años).

Iba a jugar fútbol con mis papás; mi papá jugaba conmigo y mi mamá se quedaba sentada mirando. Ella no sabe, creo que podría aprender. Le dice que vayan y ella dice que no, creo que es porque no sabe $(H, 14$ años).

Según lo expresado por los jóvenes, la diversión se presenta como gran motivador para hacer AF. Para los jóvenes, esta práctica representa una oportunidad para salir de la rutina y experimentar adrenalina y sensaciones de alegría. En algunos casos, estos momentos fueron compartidos con amigos, pero con mayor frecuencia mencionaron sensaciones personales.

[...] me llama mucho la atención andar en patines; se siente uno volando. Toca estar agachada, uno siente 
que se va a caer, pero se siente muy chévere (M, 14 años).

[...] me gusta, los disfruto [los momentos en que realiza la actividad física], me dan ganas de desmayarme, no importa que eso pase (H, 13 años).

Los adolescentes destacan que la AF proporciona varios beneficios para la salud, como el fortalecimiento de la salud física y mental, y ayuda a prevenir enfermedades. Los adolescentes enfatizan que la $\mathrm{AF}$ es un medio que puede proteger del desarrollo de enfermedades a corto y largo plazo. También ven beneficios positivos para la salud, como perder grasa, ganar masa muscular, mantener el peso corporal y la apariencia física. Además, asocian la práctica de la AF con un estado mental renovado, mayor vitalidad y menos estrés.

Alarga la vida; el que no hace actividad física es más propenso a sufrir una enfermedad. El que está en actividad física tiene más vitalidad, no se enferman (H, 14 años).

La salud; uno no se muere tan rápido, no se enferma uno casi (H, 16 años).

[...] mejora el ritmo cardiaco, ayuda a relajar el estrés, a mejorar la mente. En mí, mejora el estrés y en lo físico (M, 14 años).

Cuando uno hace ejercicio, se crece el músculo, tiene mejor salud y mejora la fuerza (H, 14 años).

En mi caso, yo era flaquita y me subí de peso; entonces, para mí es como una ayuda, algo mejor para la salud. Cuando esté más grande, sé que me va a servir mucho más (M, 14 años).

Los adolescentes reconocen la influencia de los padres en la práctica de la AF. Esto es consistente con su rol de acompañarlos, caracterizado por brindar apoyo y confianza. Se encontró que los padres se convierten en los principales promotores de esta práctica en los adolescentes, ellos son quienes los incitan a mantenerse al día con la disciplina, el esfuerzo y la puntualidad en las actividades.

Mi papá, a veces nos vemos y me dice que haga deporte. Él tiene buen físico, él me quiere meter al gimnasio. Él siempre ha sido deportista, le gusta caminar, lo lleva en la sangre (M, 15 años).

[...] cuando yo voy a entrar a un deporte, a mi mamá le gusta que, si lo comencé, debo terminarlo; no le gusta que este faltando o que vaya un día sí, un día no. Ella me apoya cuando entro a un deporte o cuando practico alguna actividad física (M, 14 años).
El entorno alrededor de las viviendas de los participantes ofrecía espacios recreativos, como parques, canchas deportivas y gimnasios al aire libre. Estos tienen una influencia positiva en la intención de los adolescentes de practicar la AF en espacios al aire libre, naturales e inclusivos.

El parque es adecuado para lanzar el balón, es para todas las personas. Caminos en piedra y con árboles. Como voy de noche, como a las 8:30 p. m., tarde, está desocupado (H, 14 años).

[...] en mi barrio sí hay espacio: hay un gimnasio, hay tres canchas y el barrio es grande como para salir a trotar o a correr, o cosas así por el estilo (M, 13 años).

\section{Barreras}

Se evidenció que, así como existen oportunidades para la práctica de la AF en aspectos como la compañía y el apoyo de los padres, estos también fueron determinantes en la no práctica, es decir, pueden ser una barrera, ya sea por experiencias con otros miembros del hogar o por falta de tiempo para el acompañamiento.

Mi papá es callado, no se expresa casi. A mi mamá no le gusta, porque dice que eso es para borrachos. A nosotros no nos ha apoyado, porque mi hermano toma mucho (H, 16 años).

En mi caso, mi papá no me deja, porque tengo que hacer tareas o porque estoy enferma. Por lo general, siempre me deja, siempre y cuando vaya la mamá de mi amiga (M, 14 años).

Los jóvenes mencionan que la falta de tiempo debido a las asignaciones escolares es una de las razones fundamentales para no participar en la AF. Esta misma razón es también la principal justificación para abandonar sus rutinas de ejercicio físico.

No alcanzaba a hacer las tareas. Mis padres tenían razón, no me alcanzaba el tiempo. Cuando dejé de practicar, mi rendimiento académico aumentó (H, 14 años).

[...] me quedaba pesado para la escuela [...] me recogían a las 7 de la noche y llegaba a casa a las 10 , y yo madrugaba al otro día; me quedaba pesado. Luego cogí los sábados y domingos, pero empecé a estudiar inglés y también me tocaba madrugar (M, 14 años).

Aunque los adolescentes reconocen que en sus vecindarios hay espacios que facilitan la AF, también identifican el hecho de que no están disponibles todo el tiempo y las condiciones de seguridad no son óptimas.

[...] en mi barrio hay gimnasio, pero toca pagar; hay tres maquinitas, pero están ya todas dañadas (M, 15 años). 
[...] no hago actividad física allá, porque hay muchos $\tilde{n}_{\operatorname{eros}}^{\dagger}$ (M, 14 años).

Finalmente, aunque la escuela es el lugar principal para practicar AF, los jóvenes consideran que el espacio y las instalaciones no son adecuados. Argumentan que a veces el uso de estos espacios está restringido y hay una limitación en el tiempo para hacer uso de ellos fuera de las clases de educación física.

No, porque allá solo hay una cancha y en los salones no se puede, el ruido no deja, en el aula máxima no cabemos todos. La cancha es muy poquito espacio ( $\mathrm{M}, 12$ años).

[...] porque el colegio no tiene las instalaciones suficientes para los estudiantes; el espacio es muy reducido y no hay oportunidad para que se desarrollen esos ejercicios y deportes (M, 13 años).

\section{Discusión}

Este estudio utilizó un enfoque cualitativo para describir la percepción de los adolescentes sobre facilitadores y barreras de la AF, adolescentes que viven en comunidades de ingresos medios y bajos, con el propósito de ampliar la comprensión de los determinantes de la práctica de la AF en esta población.

Hallazgos previos han enfatizado el uso de términos como "diversión" o "juego", sobre "actividad física" o "ejercicio", en poblaciones jóvenes, mediante juegos al aire libre y juegos no estructurados y recreativos como oportunidades deseables [21].

Se sugiere revisar estrategias comunitarias para fomentar el diálogo y la confianza entre los vecinos y, en consecuencia, promover el juego libre y activo [17].

Con respecto a los adolescentes y la AF en el tiempo libre, algunos estudios indican que las principales razones para su realización incluyen el entretenimiento, la ocupación del tiempo libre, mantenerse en forma, beneficios para la salud y el disfrute que la actividad les brinda [22]; asimismo, es deseable la práctica espontánea de AF, dado que al hacerse en el transcurso del día va acumulándose [23].

En un marco más integrador, es también importante la comprensión y la acción desde un modelo socioecológico [24], en tanto que la visión es más holística y complementa la comprensión de la práctica de AF.

Un estudio de 2019 muestra que las barreras y los facilitadores están organizados en factores motivadores y limitantes percibidos, conciencia de AF y limitaciones de tiempo percibidas, apoyo social, normas culturales y de género, y acceso a oportunidades [20].

En nuestro análisis, el concepto de AF de los adolescentes se asoció con los deportes y las actividades de la vida diaria (salir al aire libre, saltar o trotar). Entre los temas motivadores dominantes para emprender AF identificamos la recreación, la diversión, la mejora de la salud física y mental, perder grasa, ganar masa muscular, mantener el peso corporal y la forma física, y evitar las enfermedades a corto y largo plazo. Esto es consistente con los motivadores de los niños y las niñas adolescentes informados en una revisión de estudios cualitativos, que enunció razones como mantener la forma física y el control del peso corporal $[25,26]$.

También asociaron la práctica de la AF con un estado mental renovado, mayor vitalidad y disminución del estrés. Esta percepción se ha demostrado claramente en adolescentes que mantuvieron el compromiso con la $\mathrm{AF}$ a los 10 años de seguimiento y mostró un riesgo significativamente menor de enfermedad cardiovascular y mejor salud mental [27].

Por otro lado, la principal barrera para la $\mathrm{AF}$ mencionada en este estudio fue la falta de tiempo. Aunque los adolescentes comentaron opciones para realizar $\mathrm{AF}$, perdió su importancia si interfería o se percibió que interfería con las actividades escolares. Esto también se observó en estudiantes universitarios [28,29], lo que sugiere que este comportamiento persiste.

En el presente estudio, la participación en la AF en general se limitaba a las clases de educación física, y solo una pequeña proporción de adolescentes participaban en actividades extracurriculares. Esto sugiere que la escuela podría ser el punto de convergencia más importante desde el cual enfocar los esfuerzos para promover un aumento de la AF.

Los adolescentes perciben su vecindario y entorno como barreras o facilitadores de la AF. Varias características del entorno del vecindario y la escuela se vincularon con la AF (accesibilidad de los espacios y equipos, estética del espacio y los equipos, y problemas de seguridad). Nuestros hallazgos se alinean con estudios anteriores que examinan las percepciones de los jóvenes sobre el medio ambiente como determinantes de la AF. Un estudio realizado en Estados Unidos, en el que participaron 33 estudiantes en grupos focales, también identificó la accesibilidad, la estética y la seguridad como oportunidades y barreras clave para hacer AF [30]. Así mismo, en una muestra de adolescentes canadienses, la disponibilidad de recursos y las preocupaciones de seguridad igualmente fueron barreras y facilitadores clave [31]. Además, en poblaciones de bajo estatus

Aféresis de "compañero". Se usa en Colombia principalmente para designar a personas de la calle, gamines, o jóvenes que se identifican con un tipo social, una de cuyas características que se les atribuye es ser amenazantes, peligrosos. 
socioeconómico similares a las observadas en el presente estudio, los adolescentes informan que la falta o las malas condiciones y el diseño de las infraestructuras constituyen una barrera para la práctica de la AF [26]. Esto sugiere la necesidad de una transformación del espacio más inclusiva, así como la expansión de opciones seguras, gratuitas y estéticamente agradables.

Las preocupaciones de seguridad, incluidos el espacio físico y la presencia de pandillas o comportamientos amenazadores, crean barreras para el acceso a lugares para estar físicamente activos, lo que hace que los miembros de la familia acompañantes sean importantes para facilitar la AF [32].

Los jóvenes parecen más dispuestos a tolerar entornos y equipamiento menos atractivos cuando están con sus compañeros; en contraste, la amistad y el apoyo de los compañeros influencia la actividad actual y es sugerida por los adolescentes para promover la participación en AF [33]. Esto es evidente desde la infancia; Glenn et al. (2013) encontraron que los amigos y los hermanos eran los compañeros de juego preferidos en los niños [34].

En contraste, en nuestro estudio, los padres, en especial del mismo sexo, fueron los principales compañeros de los adolescentes en el tiempo libre. Los padres se convirtieron en los primordiales promotores de esta práctica en adolescentes, sugiriendo una oportunidad, pero también una posible barrera significativa para la AF en el contexto actual.

En común con nuestros hallazgos, una revisión observó que, específicamente en adolescentes de condición socioeconómica baja, la práctica de la AF estaba influenciada por miembros de la familia físicamente inactivos [26]. Como complemento, esta situación se evidencia en grupos de edad más pequeños (10-11 años), donde los padres median como facilitadores significativos (es decir, alientan) y como barrera (es decir, restringen la participación) [34]. Esta población tiene un nivel de AF más bajo que sus contrapartes de estratos socioeconómicos más altos, debido a factores sociales, individuales y ambientales. Por lo tanto, la mejor comprensión de las barreras y los facilitadores es útil para informar el diseño de intervenciones efectivas que promuevan intervenciones en estas poblaciones, que, además, son más vulnerables a enfermedades crónicas [35].

En este punto, también es importante explorar el entorno hogar, que ha demostrado mejorar o limitar las oportunidades para el comportamiento sedentario de los niños (9 a 13 años) y la $\mathrm{AF}$, sin perder de vista que el hogar es un espacio dinámico que puede cambiar y está conformado, en gran medida, por la familia que vive en él, lo que la diferencia de otros entornos, como la escuela [36].
El estudio tiene una serie de limitaciones, incluida la cantidad de participantes y el hecho de abordar solo una escuela del sector oficial, por lo que los hallazgos deben confirmarse en muestras más grandes, buscando la participación de diferentes conglomerados, la diferenciación de entornos como el hogar y la contextualización a la luz de modelos socioecológicos.

Otras limitaciones son el hecho de que los padres no fueron entrevistados y la ausencia de triangulación de información entre el componente cualitativo y cuantitativo del estudio SIMAC.

No obstante, este es el primer abordaje cualitativo basado en el conocimiento personal y la experiencia de los adolescentes en el municipio de Piedecuesta, con respecto a la práctica de la AF.

\section{Conclusiones}

Los resultados aquí presentados mostraron una percepción positiva de los adolescentes con respecto a la $\mathrm{AF}$; reconocieron sus beneficios y la importancia de la participación, y los relacionaron con sentimientos de alegría, salud y bienestar mental, y enfatizaron en la importancia de la AF como estrategia de protección contra las enfermedades a corto y largo plazo. También percibieron beneficios positivos para la salud física, como la pérdida de grasa, ganar masa muscular, mantener el peso corporal y mejorar la apariencia del cuerpo. Sin embargo, el compromiso de la AF estuvo limitado por factores externos, incluida la falta de tiempo percibida, instalaciones inadecuadas y ausencia de apoyo de los padres.

La escuela y el vecindario son valiosos puntos de reunión y convergencia para la práctica de la $\mathrm{AF}$, entornos $\mathrm{y}$ facilitadores potenciales, lo que sugiere fortalecer la práctica curricular, y transformar espacios inclusivos y expandir opciones seguras y gratuitas. Debido a la significativa influencia de los padres, sugerimos que los enfoques para mejorar los niveles de AF deben examinar el potencial de las intervenciones que incluyen al padre que acompaña al niño o niña en el mismo espacio.

Para lograr intervenciones exitosas que generen cambios medibles en el comportamiento, es necesario comprender los factores clave que influyen en la práctica de la AF; el conocimiento de estos cumple un papel importante en el diseño de intervenciones y estrategias.

\section{Conflicto de interés}

Dejamos constancia que las afirmaciones vertidas en este manuscrito son de exclusiva responsabilidad de los autores y que no existe ningún conflicto de interés de parte de ellos. 


\section{Financiación}

El Estudio SIMAC fue financiado mediante la convocatoria 657-2014 para Proyectos de Ciencia, Tecnología e Innovación en Salud 2014, ejecutado por la FOSCAL, por medio del contrato 645-2014, código 651765741093.

\section{Declaración de responsabilidad}

Declaramos que los puntos de vista expresados son responsabilidad de los autores y no de las instituciones en las que trabajamos o de la fuente de financiación.

\section{Declaración de autoría}

Todos los autores realizaron contribuciones significativas a la concepción y el diseño del manuscrito. Johanna Otero y Diana Delgado participaron en la recolección, el procesamiento y el análisis de datos. Johanna Otero, Diana Delgado, Sandra Rueda y Mónica Amador participaron en la redacción del contenido del artículo, la cual fue revisada de forma crítica, corregida y aprobada por Daniel Cohen, Paul Camacho y Patricio López-Jaramillo. Todos los autores asumen la responsabilidad frente a todos los aspectos del manuscrito, para garantizar que los asuntos relativos a la exactitud o integridad de cualquier parte de este serán adecuadamente investigados y resueltos.

\section{Referencias}

1. López-Jaramillo P, Silva SY, Rodriguez-Salamanca N, et al Are nutrition-induced epigenetic changes the link between socioeconomic pathology and cardiovascular diseases? Am J Ther. 2008;15(4):362-72.

2. Bauman AE, Reis RS, Sallis JF, et al. Correlates of physical activity: Why are some people physically active and others not? Lancet 2012; 380(9838):258-71. DOI: https://doi.org/10.1016/ S0140-6736(12)60735-1

3. Skinner AC, Perrin EM, Moss LA, et al. Cardiometabolic risks and severity of obesity in children and young adults. N Engl J Med. 2015;373(14):1307-17.

4. Yáñez-Silva A, Hespanhol JE, Gómez R, et al. Valoración de la actividad física en adolescentes escolares por medio de cuestionario. Rev Chil Nutr. 2014;41(4):360-6. DOI: http:// dx.doi.org/10.4067/S0717-75182014000400003

5. World Health Organization (WHO). Political Declaration of the High-level Meeting of the General Assembly on the Prevention and Control of Non-communicable Diseases. 2011. Geneva: WHO.

6. World Health Organization. Physical activity: Fact sheet 385 [internet]; 2015 [citado 2020 feb. 25]. Disponible en: http://www. who.int/mediacentre/factsheets/fs385/en/.

7. American Heart Association. How Can I Help My Child Be More Physically Active [internet]; 2018 [citado 2020 feb. 25] Disponible en: http://www.heart.org/HEARTORG/HealthyLiving/
HealthyKids/ActivitiesforKids/The-AHAs-Recommendationsfor-Physical-Activity-in-Children_UCM_304053_Article.jsp\#. WW0Y59M1 GI

8. Hallal PC, Andersen LB, Bull FC, et al. Global physical activity levels: Surveillance progress, pitfalls, and prospects. Lancet. 2012;380(9838):247-57. DOI: https://doi.org/10.1016/S01406736(12)60646-1

9. Ortega FB, Konstabel K, Pasquali E, et al. Objectively measured physical activity and sedentary time during childhood, adolescence and young adulthood: A cohort study. PLoS ONE. 2013;8(4):e60871. DOI: https://doi.org/10.1371/journal. pone. 0060871

10. Instituto Colombiano de Bienestar Familiar. Encuesta nacional de la situación nutricional en Colombia. Estado nutricional por indicadores antropométricos [internet]; 2005 [citado 2020 feb. 25]. Disponible en: https://www.icbf.gov.co/bienestar/nutricion/ encuesta-nacional-situacion-nutricional\#ensin 1

11. Secretaría de Salud de Santander, Observatorio de Salud Pública de Santander. Factores de riesgo para enfermedades crónicas en Santander, método STEPwise. Bucaramanga: 11. Secretaría de Salud de Santander, Observatorio de Salud Pública de Santander [internet]. 2011 [citado 2020 feb. 25]. Disponible en: http://www. who.int/chp/steps/2010_STEPS_Survey_Colombia.pdf

12. Telama $\mathrm{R}$, Yang $\mathrm{X}$, Viikari J, et al. Physical activity from childhood to adulthood: A 21-year tracking study. Am J Prev Med. 2005;28(3):267-73. DOI: https://doi.org/10.1016/j. amepre.2004.12.003

13. Burton NW, Turrell G, Oldenburg B, et al. The relative contributions of psychological, social, and environmental variables to explain participation in walking, moderate-, and vigorous-intensity leisure-time physical activity. J Phys Activ Health. 2005;2(2):18196. DOI: https://doi.org/10.1123/jpah.2.2.181

14. Ruiz F, García ME, Díaz A. Análisis de las motivaciones de práctica de actividad física y de abandono deportivo en la ciudad de La Habana (Cuba). Anales de Psicología. 2007;23(1):152-66.

15. Muñoz-Daw M, Muñoz-Duarte M, De La Torre-Díaz M, et al. Motivos para la práctica de actividad física recreativa e inactividad en la población de Chihuahua (México). Nutr Clín Diet Hosp. 2016;36(1):10-16. DOI: https://doi.org/10.12873/361muñozdaw

16. Verhoeven H, Simons D, Van Dyck D, et al. Psychosocial and environmental correlates of walking, cycling, public transport and passive transport to various destinations in flemish older adolescents. PLoS ONE. 2016;11(1):e0147128. DOI: https://oi. org/10.1371/journal.pone.0147128

17. Lee H, Tamminen K, Clark A, et al. A meta-study of qualitative research examining determinants of children's independent active free play. Int J Behav Nutr Phys Act. 2015;12:1-12. DOI: https:// doi.org/10.1186/s12966-015-0165-9

18. Lu W, McKyer EL, Lee C, et al. Perceived barriers to children's active commuting to school: A systematic review of empirical, methodological and theoretical evidence. Int J Behav Nutr Phys Act. 2014;11:1-20. https://doi.org/10.1186/s12966-014-0140-x.

19. Ramírez-Vélez R, Beltrán CA, Correa-Bautista JE, et al. Factors associated with active commuting to school by bicycle from Bogotá, Colombia: The FUPRECOL study. Ital J Pediatr. 2016;42(1):97. DOI: https://doi.org/10.1186/s13052-016-0304-1

20. Abdelghaffar E, Hicham EK, Siham B, et al. Perspectives of adolescents, parents, and teachers on barriers and facilitators of physical activity among school-age adolescents: A qualitative analysis. Environ Health Prev Med. 2019;24:1-13. DOI: https:// doi.org/10.1186/s12199-019-0775-y 
21. Ross SE, Francis LA. Physical activity perceptions, context, barriers, and facilitators from a Hispanic child's perspective. Int J Qual Studi Health Well-being. 2016;11(1):1-11. DOI: https://doi. org/10.3402/qhw.v11.31949

22. Rodríguez-Romo G, Boned-Pascual C, Garrido-Muñoz M. Motivos y barreras para hacer ejercicio y practicar deportes en Madrid. Rev Pan Salud Pública. 2009;26(3):244-54. DOI: https:// doi.org/10.1590/s1020-49892009000900009

23. Jonsson L, Berg C, Larsson C, et al. Facilitators of physical activity: Voices of adolescents in a disadvantaged community. Int J Environ Res Public Health. 2017;14(8):1-13. DOI: https://doi. org/10.3390/ijerph14080839

24. Carson RL, Castelli DM, Beighle A, et al. School-based physical activity promotion: A conceptual framework for research and practice. Child Obes. 2014;10(2):100-6. DOI: https://doi. org/10.1089/chi.2013.0134.

25. Allender S, Cowbum G, Foster C. Understanding participation in sport and physical activity among children and adults: A review of qualitative studies. Health Educ Res. 2006;21(6):826-35. DOI: https://doi.org/10.1093/her/cyl063

26. Martins J, Marques A, Sarmento H, et al. Adolescents' perspectives on the barriers and facilitators of physical activity: a systematic review of qualitative studies. Health Educ Res. 2015;30(5):74255. DOI: https://doi.org/10.1093/her/cyv042

27. Rangul V, Bauman A, Holmen TL, et al. Is physical activity maintenance from adolescence to young adulthood associated with reduced CVD risk factors, improved mental health and satisfaction with life: The HUNT Study, Norway. Int J Behav Nutr Phys Act [internet]. 2012 [citado 2020 feb. 25]; 9:1-11. Disponible en: https:// ijbnpa.biomedcentral.com/articles/10.1186/1479-5868-9-144. DOI: https://doi.org/10.1186/1479-5868-9-144

28. Ashton LM, Hutchesson MJ, Rollo ME, et al. Young adult males' motivators and perceived barriers towards eating healthily and being active: A qualitative study. Int J Behav Nutr Phys Act. 2015;12(1):1-10. DOI: https://doi.org/10.1186/s12966-015-0257-6

29. Walsh JR, White AA, Greaney ML. Using focus groups to identify factors affecting healthy weight maintenance in college men. Nutr Res. 2009;29(6):371-8. DOI: https://doi.org/10.1016/j. nutres.2009.04.002

30. Smith AL, Troped PJ, McDonough MH, et al. Youth perceptions of how neighborhood physical environment and peers affect physical activity: A focus group study. Int J Behav Nutr Phys Act. 2015;12(1):1-9. DOI: https://doi.org/10.1186/s12966-015-0246-9

31. Holt NL, Cunningham CT, Sehn ZL, et al. Neighborhood physical activity opportunities for inner-city children and youth. Health Place. 2009;15(4):1022-8. DOI: https://doi.org/10.1016/j. healthplace.2009.04.002.

32. Salmon J, Spence JC, Timperio A, et al. Living environments. En: Smith AL, Biddle S., editores. Youth Physical Activity and Sedentary Behavior: Challenges and Solutions, United States: Human Kinetics; 2008. pp. 403-28.

33. Carlin A, Murphy MH, Gallagher AM. Current influences and approaches to promote future physical activity in 11-13 year olds: A focus group study. BMC Public Health. 2015;15(1):1-12. DOI: https://doi.org/10.1186/s12889-015-2601-9

34. Glenn NM, Knight CJ, Holt NL, et al. Meanings of play among children. Childhood. 2013;20(2):185-99. DOI: https://doi. org/10.1177/0907568212454751

35. Palafox B, McKee M, Balabanova D, et al. Wealth and cardiovascular health: A cross-sectional study of wealthrelated inequalities in the awareness, treatment and control of hypertension in high-, middle- and low-income countries. Int $\mathrm{J}$ Equity Health. 2016;15(1):1-14. DOI: https://doi.org/10.1186/ s12939-016-0478-6

36. Maitland C, Stratton G, Foster S, et al. The Dynamic Family Home: A qualitative exploration of physical environmental influences on children's sedentary behaviour and physical activity within the home space. Int J Behav Nutr Phys Act. 2014;11:1-12. DOI: https://doi.org/10.1186/s12966-014-0157-1 